Embryo quality is the main factor affecting cumulative live birth rate after elective single embryo transfer in fresh stimulation cycles

\title{
Niinimaki, Maarit
}

2015-11

Niinimaki , M , Veleva , Z \& Martikainen , H 2015 , ' Embryo quality is the main factor affecting cumulative live birth rate after elective single embryo transfer in fresh stimulation cycles ' , European Journal of Obstetrics, and Gynecology ,and Reproductive Biology, vol. 194 , pp. 131-135 . https://doi.org/10.1016/j.ejogrb.2015.08.031

http://hdl.handle.net/10138/223834

https://doi.org/10.1016/j.ejogrb.2015.08.031

publishedVersion

Downloaded from Helda, University of Helsinki institutional repository.

This is an electronic reprint of the original article.

This reprint may differ from the original in pagination and typographic detail.

Please cite the original version. 


\title{
Embryo quality is the main factor affecting cumulative live birth rate after elective single embryo transfer in fresh stimulation cycles
}

\author{
Maarit Niinimäki ${ }^{\mathrm{a}, \mathrm{b}, *}$, Zdravka Veleva $^{\mathrm{c}}$, Hannu Martikainen ${ }^{\mathrm{a}, \mathrm{b}}$ \\ a Department of Obstetrics and Gynecology, Oulu University Hospital, 90029 Oulu, Finland \\ ${ }^{\mathrm{b}}$ University of Oulu, PEDEGO Research Unit, Oulu and Medical Research Center, Oulu, Finland \\ ${ }^{\mathrm{c}}$ Department of Obstetrics and Gynecology, University of Helsinki and Helsinki University Central Hospital, 00029 Helsinki, Finland
}

\section{A R T I C L E IN F O}

\section{Article history:}

Received 8 June 2015

Received in revised form 13 August 2015

Accepted 19 August 2015

\section{Keywords:}

Elective single embryo transfer

Cumulative live birth rate

In vitro fertilization

\begin{abstract}
A B S T R A C T
Objective: The study was aimed to evaluate which factors affect the cumulative live birth rate after elective single embryo transfer in women younger than 36 years. Additionally, number of children in women with more than one delivery per ovum pick-up after fresh elective single embryo transfer and subsequent frozen embryo transfers was assessed.

Study design: Retrospective cohort study analysing data of a university hospital's infertility clinic in 2001-2010. A total of 739 IVF/ICSI cycles with elective single embryo transfer were included. Analyses were made per ovum pick-up including fresh and subsequent frozen embryo transfers. Factors affecting cumulative live birth rates were examined in uni- and multivariate analyses. A secondary endpoint was the number of children born after all treatments.

Results: In the fresh cycles, the live birth rate was $29.2 \%$ and the cumulative live birth rate was $51.3 \%$, with a twin rate of $3.4 \%$. In the multivariate analysis, having two (odds ratio (OR) 1.73; 95\% confidence interval (CI) 1.12-2.67) or $\geq 3$ top embryos (OR 2.66; 95\% CI 1.79-3.95) was associated with higher odds for live birth after fresh and frozen embryo cycles. Age, body mass index, duration of infertility, diagnosis or total gonadotropin dose were not associated with the cumulative live birth rate. In cycles with one top embryo, the cumulative live birth rate was $40.2 \%$, whereas it was $64.1 \%$ in those with at least three top embryos. Of women who had a live birth in the fresh cycle, $20.4 \%$ had more than one child after all frozen embryo transfers. Among women with three or more top embryos after ovum pick-up, $16.1 \%$ gave birth to more than one child.

Conclusion: The cumulative live birth rate in this age group varies from $40 \%$ to $64 \%$ and is dependent on the quality of embryos. Women with three or more top embryos have good chance of having more than one child per ovum pick-up without elevated risk of multiple pregnancies.
\end{abstract}

(c) 2015 Elsevier Ireland Ltd. All rights reserved.

\section{Introduction}

In in vitro fertilisation (IVF) or intracytoplasmic sperm injection (ICSI), elective single embryo transfer (eSET) is an effective method to decrease multiple births which cause increased maternal and perinatal morbidity and mortality [1]. Largely due to the introduction of eSET, the multiple delivery rate per embryo transfer has declined steadily in Europe from 26.9\% in 2000 to $19.2 \%$ in 2010 [1]. eSET is widely practiced in the Nordic countries. Accordingly,

\footnotetext{
* Corresponding author at: Department of Obstetrics and Gynecology, Oulu University Hospital, P.O. Box 24, 90029 Oulu, Finland. Tel.: +358 503371922

E-mail address: maarit.niinimaki@oulu.fi (M. Niinimäki).
}

statistics from 2011 show a low proportion of multiple births after IVF/ICSI - from 5.1\% (Sweden) to $16.5 \%$ (Denmark) [2].

Embryo freezing is an essential component of eSET policy. Frozen embryo transfer (FET) cycles account for $31.8 \%$ of all assisted reproduction technology (ARTs) in the Nordic countries. The rate is highest in Finland (45.1\%) [2]. Previous studies have confirmed that the cumulative pregnancy and live birth rates (cLBR) evaluating the outcome of ovum pick-up (OPU) and all following FETs indicate the real efficiency of ART and should be used when compared with other transfer policies $[3,4]$. eSET, combined with an effective cryopreservation programme, has been shown to result in a high cumulative pregnancy rate (44-62\%) per oocyte retrieval [5-8]. The success in IVF treatment has been shown to have association with morphological parameters of the embryo [9]. On the other hand, a recent study indicated that embryo quality has no effect on maternal or 
neonatal outcomes in SET cycles [10]. There are few studies on factors affecting cumulative outcomes in eSET cycles.

According to earlier analyses, eSET diminished the costs in the general IVF/ICSI population [11], especially among women aged 36 years or less [12]. Studies comparing cLBR in eSET and double embryo transfer (DET) cycles in older women also suggest eSET is a viable treatment option [13,14]. Currently, eSET is more commonly applied to women younger than 36 years [1]. eSET has been suggested as a treatment of choice in this age group, even in countries with low eSET use [12,15].

The primary aim of the present study was to investigate the factors that affect cLBR after eSET in a cohort of women younger than 36 years. The results could be useful in counselling couples undergoing infertility treatment. A secondary aim was to analyse how many children were born in women with more than one delivery after single IVF stimulation, including deliveries after eSET in fresh cycle and subsequent FET cycles. This aspect has not been studied previously, although from the point of view of the couples information on the possible number of children gained with a single stimulated cycle is undoubtedly important.

\section{Materials and methods}

This retrospective cohort study population consisted of all first IVF/ICSI treatments with eSET performed in the fresh cycle on day 2 after ovum pick-up (ultrasound-guided oocyte aspiration under intravenous sedation) in the time period 2001-2010 in Oulu University Hospital, Department of Obstetrics and Gynaecology, Oulu, Finland. eSET was defined as a treatment in which one embryo was transferred in the fresh cycle and at least one embryo was frozen. All subjects were younger than 36 years at the time of OPU. The analyses were made per treatment (OPU). The study consisted only of couples treated with their own gametes. Cumulative analyses were carried out by identifying FET cycles following OPU using the identification code in the Babe ${ }^{\circledR}$ database for ART patients (Cleodora Software, Portugal). In the FET cycles one or two embryos were transferred at the time. As one of the key issues was to determine the impact of the embryo quality on the success rate, women with missing data on the quality of embryos were excluded. Ovarian stimulation was mainly performed using the long GnRH agonist protocol (93.0\%, 688 cycles) or the GnRH antagonist protocol (3.5\%, 26 cycles). In 25 cycles (3.5\%), other stimulation protocols were used. The starting gonadotrophin dose was determined according to the patient's age, BMI, antral follicular count in the baseline ultrasonographic scan and the outcome of previous infertility treatments. A top quality embryo had $4-5$ evenly sized cells and $<20 \%$ of fragmentation [9]. Embryos not transferred in the fresh cycle were frozen on the day of embryo transfer, using a slow freezing protocol.

Data on the woman's age at the time of IVF or ICSI treatment, body mass index (BMI) $\left(\mathrm{kg} / \mathrm{m}^{2}\right)$, duration of infertility at the time of IVF or ICSI (years), main cause of infertility, number of oocytes

Table 1

Characteristics, $N(\%)$.

\begin{tabular}{|c|c|c|c|c|c|}
\hline & \multicolumn{5}{|c|}{ By the number of top embryos } \\
\hline & All, $N=739$ & 1 & 2 & 3 or more & $p$ Value \\
\hline \multicolumn{6}{|l|}{ Age (years) } \\
\hline Younger than 30 & $354(47.9)$ & $132(37.3)$ & $80(22.6)$ & $142(40.1)$ & 0.22 \\
\hline $30-35$ & $385(52.1)$ & $154(40.0)$ & $100(26.0)$ & $131(34.0)$ & \\
\hline \multicolumn{6}{|l|}{$B M I\left(k g / m^{2}\right)^{\mathrm{a}}$} \\
\hline$<20.0$ & $97(13.3)$ & $42(43.3)$ & $22(22.7)$ & $33(34.0)$ & 0.06 \\
\hline $20.0-24.9$ & $432(59.0)$ & $171(39.6)$ & $100(23.1)$ & $161(37.3)$ & \\
\hline $25.0-29.9$ & $140(19.1)$ & $51(36.4)$ & $32(22.9)$ & $57(40.7)$ & \\
\hline $30.0-34.9$ & $51(7.0)$ & $17(33.3)$ & $15(29.4)$ & $19(37.3)$ & \\
\hline 35.0 or more & $12(1.6)$ & $1(8.3)$ & $8(66.7)$ & $3(25.0)$ & \\
\hline \multicolumn{6}{|l|}{ Duration of infertility (years) ${ }^{\mathrm{b}}$} \\
\hline Less than 1 & $9(1.5)$ & $1(0.4)$ & $1(0.7)$ & $7(3.0)$ & 0.04 \\
\hline $1-2$ years & $201(32.6)$ & $72(30.6)$ & $42(28.0)$ & $87(37.5)$ & \\
\hline $3-4$ years & $247(40.0)$ & $105(44.7)$ & $63(42.0)$ & $79(34.1)$ & \\
\hline 5 years or more & $160(25.9)$ & $57(24.3)$ & $44(29.3)$ & $59(25.4)$ & \\
\hline \multicolumn{6}{|l|}{ Main diagnosis } \\
\hline Endometriosis & $140(18.9)$ & $61(21.3)$ & $29(16.1)$ & $50(18.3)$ & 0.09 \\
\hline Hormonal & $69(9.3)$ & $16(5.6)$ & $21(11.7)$ & $32(11.7)$ & \\
\hline Tubal & $89(12.0)$ & $38(13.3)$ & $14(7.8)$ & $37(13.6)$ & \\
\hline Male & $232(31.4)$ & $90(31.5)$ & $57(31.7)$ & $85(31.1)$ & \\
\hline Unexplained & $161(21.8)$ & $59(20.6)$ & $45(25.0)$ & $57(20.9)$ & \\
\hline Multiple \& other reasons & $48(6.5)$ & $22(7.7)$ & $14(7.8)$ & $12(4.4)$ & \\
\hline \multicolumn{6}{|l|}{ Type of ART } \\
\hline IVF & $433(58.6)$ & $169(59.1)$ & $103(57.2)$ & $161(59.0)$ & 0.92 \\
\hline ICSI or IVF + ICSI & $306(41.4)$ & $117(40.9)$ & $77(42.8)$ & $112(41.0)$ & \\
\hline Gonadotropin dose IU (mean +SD) & $1920(659)$ & $2051(776)$ & $1865(511)$ & $1819(586)$ & $<0.001$ \\
\hline \multicolumn{6}{|l|}{ Number of oocytes } \\
\hline Less than 10 & $216(29.2)$ & $124(43.4)$ & $53(29.4)$ & $39(14.3)$ & $<0.001$ \\
\hline $10-20$ & $419(56.7)$ & $140(49.0)$ & $104(57.8)$ & $175(64.1)$ & \\
\hline More than 20 & $104(14.1)$ & $22(7.7)$ & $23(12.8)$ & $59(21.6)$ & \\
\hline \multicolumn{6}{|l|}{ Number of embryos frozen } \\
\hline $1-4$ & $344(46.5)$ & $200(69.9)$ & $90(50.0)$ & $54(19.8)$ & $<0.001$ \\
\hline $5-10$ & $321(43.4)$ & $78(27.3)$ & $82(45.6)$ & $161(59.0)$ & \\
\hline More than 10 & $74(10.0)$ & $8(2.8)$ & $8(4.4)$ & $58(21.2)$ & \\
\hline
\end{tabular}

BMI, body mass index; ART, assisted reproductive technology.

IVF+ ICSI, IVF and ICSI used in 50\%/50\% of the oocytes.

a Data missing in 7 cases (0.9\%).

b Data missing in 122 cases (16.5\%). 
Table 2

Pregnancy outcomes, $N(\%)$.

\begin{tabular}{|c|c|c|c|c|c|}
\hline & \multicolumn{5}{|c|}{ By the number of top embryos } \\
\hline & All $(N=739)$ & $1(N=286)$ & $2(N=180)$ & 3 or more $(N=273)$ & $p$ Value \\
\hline Clinical pregnancy & $259(35.0)$ & $100(35.0)$ & $61(33.9)$ & $98(35.9)$ & 0.91 \\
\hline LBR/OPU & $216(29.2)$ & $82(28.7)$ & $51(28.3)$ & $83(30.4)$ & 0.88 \\
\hline cLBR/OPU & $379(51.3)$ & $115(40.2)$ & $89(49.4)$ & $175(64.1)$ & $<0.001$ \\
\hline Twins in fresh cycle & $1(0.1)$ & 1 & 0 & 0 & \\
\hline Twins in FET cycle & $12(1.6)$ & $2(0.7)$ & $3(1.7)$ & $7(2.6)$ & 0.22 \\
\hline \multicolumn{6}{|c|}{ Number of children per OPU } \\
\hline 1 & $303(41.0)$ & $99(34.6)$ & $72(40.0)$ & $132(48.4)$ & 0.23 \\
\hline 2 & $64(8.7)^{a}$ & $14(4.9)$ & $15(8.3)$ & $35(12.8)$ & \\
\hline 3 & $12(1.6)^{\mathrm{b}}$ & $2(0.7)$ & $2(1.1)$ & $8(2.9)$ & \\
\hline
\end{tabular}

LBR, live birth rate; cLBR, cumulative live birth rate; OPU, ovum pick-up; FET, frozen embryo transfer.

a Including 8 twin pairs.

b Including 4 twin pairs.

retrieved, total gonadotropin dose in international units (IU) and total number of good quality embryos were retrospectively collected. Clinical pregnancy was defined as a visible heartbeat of the foetus in a transvaginal scan in the 6th to 8th week of pregnancy, which is routinely offered to patients undergoing ART. Data on the pregnancy outcome (live birth, spontaneous abortion, ectopic pregnancy, induced abortion or stillbirth) were obtained from the Babe ${ }^{\mathbb{R}}$ database together with the number of children born at the time and data on number of children born after a single OPU and following FETs in women with more than one delivery. Ethics committee of the local hospital district (Northern Ostrobothnia Hospital District) considered that approval of the committee in not needed in local register study in which all clinical information has been conducted as a retrospective audit and the patients have not been contacted for the purpose of this study.

Table 1 shows patient characteristics and Table 2 presents the pregnancy outcomes. The different groups were compared with a chi-square test or Fisher's exact test if $>20 \%$ of parameters had a value less than 5 (duration of infertility). A Kruskal-Wallis test was performed for the statistical comparison of continuous parameters with a skewed distribution (gonadotropin dose). Logistic regression was performed for each parameter (age, BMI, duration of infertility, cause of infertility, type of ART, total dose of gonadotropin and number of good quality embryos) in a univariate analysis of cLBR (Table 3), and all the parameters in univariate analysis were then added to a multivariate analysis model. As the numbers of oocytes and frozen embryos showed dependence with

Table 3

Results of the uni- and multivariate analysis for the cLBR/OPU.

\begin{tabular}{|c|c|c|c|c|}
\hline & \multicolumn{2}{|l|}{ Univariate } & \multicolumn{2}{|l|}{ Multivariate } \\
\hline & OR $(95 \% \mathrm{CI})$ & $p$ Value & OR $(95 \% \mathrm{CI})$ & $p$ Value \\
\hline \multicolumn{5}{|l|}{ Age } \\
\hline$<30$ & 1 (ref) & & 1 (ref) & \\
\hline $30-35$ & $0.83(0.62-1.11)$ & 0.21 & $0.82(0.58-1.16)$ & 0.26 \\
\hline \multicolumn{5}{|l|}{$B M I\left(\mathrm{~kg} / \mathrm{m}^{2}\right)$} \\
\hline$<20.0$ & $1.21(0.77-1.91)$ & 0.41 & $1.35(0.80-2.26)$ & 0.26 \\
\hline $20.0-24.9$ & 1 (ref) & & 1 (ref) & \\
\hline $25.0-29.9$ & $0.72(0.49-1.05)$ & 0.09 & $0.67(0.43-1.05)$ & 0.08 \\
\hline $30.0-34.9$ & $0.72(0.40-1.29)$ & 0.27 & $0.76(0.39-1.47)$ & 0.41 \\
\hline$\geq 35.0$ & $0.63(0.20-2.00)$ & 0.43 & $0.55(0.15-2.05)$ & 0.37 \\
\hline \multicolumn{5}{|l|}{ Duration of infertility } \\
\hline Less than 1 year & $1.08(0.28-4.15)$ & 0.92 & $0.66(0.16-2.69)$ & 0.57 \\
\hline $1-2$ years & $0.99(0.66-1.52)$ & 1.00 & $0.91(0.58-1.42)$ & 0.67 \\
\hline $3-4$ years & $0.83(0.56-1.23)$ & 0.35 & $0.87(0.58-1.35)$ & 0.58 \\
\hline$>4$ years & 1 (ref) & & 1 (ref) & \\
\hline \multicolumn{5}{|l|}{ Main diagnosis } \\
\hline Endometriosis & 1 (ref) & & 1 (ref) & \\
\hline Male & $1.07(0.70-1.63)$ & 0.75 & $0.92(0.53-1.58)$ & 0.76 \\
\hline Tubal & $1.03(0.60-1.75)$ & 0.92 & $0.97(0.52-1.82)$ & 0.93 \\
\hline Hormonal & $1.13(0.63-2.01)$ & 0.69 & $0.83(0.42-1.63)$ & 0.58 \\
\hline Unexplained & $0.86(0.55-1.36)$ & 0.52 & $0.78(0.46-1.32)$ & 0.36 \\
\hline Multiple and other reasons & $0.55(0.28-1.08)$ & 0.08 & $0.52(0.22-1.19)$ & 0.12 \\
\hline \multicolumn{5}{|l|}{ Type of ART } \\
\hline IVF & 1 (ref) & & 1 (ref) & \\
\hline ICSI or IVF + ICSI & $1.10(0.82-1.47)$ & 0.54 & $1.05(0.70-1.57)$ & 0.81 \\
\hline Gonadotropin dose & $1.00(1.00-1.00)$ & 0.04 & $1.0(1.00-1.00)$ & 0.40 \\
\hline \multicolumn{5}{|c|}{ Total number of good quality embryos } \\
\hline 1 & 1 (ref) & & 1 (ref) & \\
\hline 2 & $1.45(0.99-2.19)$ & 0.05 & $1.73(1.12-2.67)$ & 0.01 \\
\hline 3 or more & $2.66(1.89-3.74)$ & $<0.001$ & $2.66(1.79-3.95)$ & $<0.001$ \\
\hline
\end{tabular}

BMI, body mass index; ART, assisted reproductive technology; ref, reference. 
the number of top embryos ( $p$ value $<0.05$ in cross tabulation in Table 1 ), they were left out of uni- and multivariate analysis (Table 3). A $p$ value less than 0.05 was considered statistically significant. Analysis was performed using the IBM Statistical Package for the Social Sciences software package, version 19.

\section{Results}

The final study population consisted of 739 women younger than 36 years who underwent eSET cycles in 2001-2010. Eleven cycles were excluded due to missing data on embryo quality. Characteristics of the fresh cycles are shown in Table 1 . The mean age of the patients was 29.6 years (range $20-35$ years). The most common causes of infertility were male factor (31.4\%), unexplained infertility (21.8\%) and endometriosis (18.9\%). BMI, the total gonadotropin dose and the number of oocytes retrieved differed between the groups.

The pregnancy outcomes are shown in Table 2. The clinical pregnancy rate in the fresh cycles was $35.0 \%$. Of these clinical pregnancies, $83.4 \%$ ended in live birth and $13.1 \%$ in miscarriage. The live birth rate in the fresh cycles was $29.2 \%$. The cLBR per OPU was $51.3 \%$. The cLBR significantly increased with a higher number of top embryos. In the OPUs with one top embryo, $40.2 \%$ had at least one live birth following a fresh cycle and all performed FET cycles. In cases with two good quality embryos, the cLBR was $49.4 \%$. In those with three or more good quality embryos, the cLBR was $64.1 \%(p<0.001)$.

In the fresh eSET cycle, one monochorionic twin pregnancy occurred. In the FET cycles altogether, 12 twin pairs were diagnosed following the transfer of two embryos. The cumulative twin rate was $3.4 \%$. In the whole cohort, 303 (41.0\%) OPUs resulted in the birth of one child, 64 (8.7\%) OPUs - in the birth of two children, including one twin pair in a fresh cycle and seven twin pairs in FET cycles, and 12 (1.6\%) OPUs resulted in the birth of three children, including four twin pairs in FET cycles. There were 217 children (46.4\%) born after the fresh transfers, and 250 children $(53.5 \%)$ were born after FET ( 467 children born altogether). In cases with a live birth in a fresh cycle $(n=216), 39(18.1 \%)$ resulted in the birth of two children and $5(2.3 \%)$ OPUs in the birth of three children when a fresh cycle and FETs were all counted.

In the univariate logistic regression (Table 3) for the cLBR per OPU gonadotropin dose and the number of top embryos were significant. All parameters were included in a multivariate analysis (Table 3). In the multivariate analysis, having two (OR 1.73; 95\% confidence interval (CI) 1.12-2.67) or three or more top embryos (OR 2.66; 95\% CI 1.79-3.95) increased the odds for cumulative live birth.

\section{Comment}

Among the parameters analysed, embryo quality was the most important determinant of the cLBR per oocyte retrieval in eSET cycles. Of women with three or more good quality embryos, a high proportion (63.8\%) achieved a live birth. These results show that well-functioning cryopreservation policy plays an important role, as $53.5 \%$ of the children were born after the transfer of thawed embryos. In the Nordic countries FET is an essential part of eSET practice and the proportion of FET cycles is high. According to Nordic statistics in 2011, the cLBR is highest in Finland (33.7\%) when compared to other Nordic countries (25.4\% in Denmark, $26.9 \%$ in Norway and $31.2 \%$ in Sweden), probably reflecting the highest number of FET cycles performed in Finland [2]. The present results are in line with the findings of a smaller Swedish study comparing cumulative live birth rates in SET or DET [16] although in their study the cumulative live birth rates were evidently lower
(34.8\% in the first fresh SET cycle and $32.2 \%$ in the second fresh cycle) than in the present study (51.3\%) [16].

The number of children born after single OPU and following FET cycles in eSET has not been reported in earlier literature. Of women having favourable response to stimulation and three or more good quality embryos, $16.1 \%$ had more than one child after the last FET treatment. A live birth in fresh eSET cycle also is a good prognostic sign, as among women having a live birth in the fresh cycle, $20.4 \%$ had more than one child after FET(s). This means that the chance of having more than one child is significant and should be included in couple's counselling information when planning eSET. As expected, the cumulative twin birth rate was low (3.4\%), which is in line with previous research $[6,14,17]$. These figures are quite reassuring, especially as a recent study showed that having IVF twins increased the risks of preterm birth, low birth weight and pre-eclampsia when compared with two IVF singleton pregnancies [18].

The present study fills in a significant gap in the understanding of infertility treatment. Cumulative data have been available in few countries such as Finland and Sweden $[3,7,8,16]$ which are the countries with higher use of frozen-thawed embryo transfer [1]. During the last decade, outcome from FET cycles has improved, as seen for example in results from the USA [19]. This progress may reflect improved cryopreservation protocols, but could also be a consequence of the increased number of eSETs enabling more good quality embryos for freezing [20]. However, cumulative results from IVF are still not always reported even though cLBR is the best measure of the real efficiency of the treatment protocol and should be more widely used.

Although the results of the study give valuable information to professionals counselling couples about eSET, some cautions should be taken into account. The main limitation of the study is that the data is retrospective and collected from the single clinic. Study subjects were also younger than the typical patients of European IVF centres. However, we believe that the results can be generalised to clinics with experience in eSET and cryopreservation.

In conclusion, our study shows that the cumulative outcome of eSET in women younger than 36 depends mostly on the embryo quality. The use of eSET strategy results in excellent take-babyhome ratios when embryo freezing is used actively. Also, according to our analyses couples with eSET have a relatively good chance of having more than one child following single OPU if the quality of embryos is good. When having three or more top embryos, every fifth woman had more than one child using embryos from the same stimulation with a low twin rate. This novel information is useful and reassuring for the patients when counselling and choosing the most appropriate approach (eSET versus double embryo transfer) in IVF.

\section{Funding}

The study was supported by the Oulu University Hospital Research Fund and the Helsinki University Central Hospital.

\section{Acknowledgement}

We thank MSc Tanja Nordström for her valuable help in the statistical analyses.

\section{References}

[1] ESHRE, 2014. http://www.eshre.eu/Guidelines-and-Legal/ART-fact-sheet.aspx [accessed 18.05.15]

[2] Nordic Fertility Society, 2014. http://www.nordicfs.org/images/2013_docs/ Nordic_IVF-2011-Haugesund_KE.pdf [accessed 18.05.15]. 
[3] Tiitinen A, Hyden-Granskog C, Gissler M. What is the most relevant standard of success in assisted reproduction? The value of cryopreservation on cumulative pregnancy rates per single oocyte retrieval should not be forgotten. Hum Reprod 2004;19:2439-41.

[4] Stillman RJ, Richter KS, Jones HW. Refuting a misguided campaign against the goal of single-embryo transfer and singleton birth in assisted reproduction. Hum Reprod 2013;28:2599-607.

[5] Vilska S, Tiitinen A, Hyden-Granskog C, Hovatta O. Elective transfer of one embryo results in an acceptable pregnancy rate and eliminates the risk of multiple birth. Hum Reprod 1999;14:2392-5.

[6] Tiitinen A, Halttunen M, Härkki P, Vuoristo P, Hyden-Granskog C. Elective single embryo transfer: the value of cryopreservation. Hum Reprod 2001:16:1140-4.

[7] Martikainen H, Orava M, Lakkakorpi J, Tuomivaara L. Day 2 elective single embryo transfer in clinical practice: better outcome in ICSI cycles. Hum Reprod 2004;19:1364-6.

[8] Thurin-Kjellberg A, Olivius C, Bergh C. Cumulative live-birth rates in a trial of single-embryo or double-embryo transfer. N Engl J Med 2009;361:1812-3.

[9] Van Royen E, Mangelschots K, De Neubourg D, et al. Characterization of a top quality embryo, a step towards single-embryo transfer. Hum Reprod 1999;14:2345-9.

[10] Oron G, Son WY, Buckett W, Tulandi T, Holzer H. The association between embryo quality and perinatal outcome of singletons born after single embryo transfers: a pilot study. Hum Reprod 2014;29:1444-51.

[11] Veleva Z, Karinen P, Tomas C, Tapanainen JS, Martikainen H. Elective single embryo transfer with cryopreservation improves the outcome and diminished the costs of IVF/ICSI. Hum Reprod 2009;24:1632-9.

[12] Scotland GS, McLernon D, Kurinczuk JJ, et al. Minimising twins in in vitro fertilization: a modeling study assessing the costs, consequences and cost-utility of elective single versus double embryo transfer over a 20 -year time horizon. BJOG 2011;118:1073-83.

[13] Veleva Z, Vilska S, Hyden-Granskog C, Tiitinen A, Tapanainen JS, Martikainen H. Elective single embryo transfer in women aged 36-39 years. Hum Reprod 2006;21:2098-102.

[14] Niinimäki M, Suikkari AM, Mäkinen S, Söderströn-Anttila V, Martikainen H Elective single embryo transfer in women aged 40-44 years. Hum Reprod 2013;28:331-5.

[15] Luke B, Brown MB, Grainger DA, et al. Practice patterns and outcomes with the use of single embryo transfer in the United States. Fertil Steril 2010;93:490-8.

[16] Lundin K, Bergh C. Cumulative impact of adding frozen-thawed cycles to single versus double fresh embryo transfers. Reprod BioMed Online 2007;15:76-82.

[17] Veleva Z, Orava M, Nuojua-Huttunen S, Tapanainen JS, Martikainen H. Factors affecting the outcome of frozen-thawed embryo transfer. Hum Reprod 2013;9(28):2425-31.

[18] Sazonova A, Källen K, Thurin-Kjellberg A, Wennerholm UB, Bergh C. Neonatal and maternal outcomes comparing women undergoing two in vitro fertilization (IVF) singleton pregnancies and women undergoing one IVF twin pregnancy. Fertil Steril 2013;99:731-7.

[19] Centers for Disease Control and Prevention, American Society for Reproductive Medicine, Society for Assister Reproductive Technology. 2011 assisted reproductive technology fertility clinic success rates report. Atlanta: U.S. Dept of Health and Human Services; 2013, Available at http://nccd.cdc.gov/DRH_ART/ Apps/FertilityClinicReport.aspx [Accessed 16.06.14].

[20] Wong KM, Mastenbroek S, Repping S. Cryopreservation of human embryos and its contribution to in vitro fertilization success rates. Fertil Steril 2014;102:19-26. 